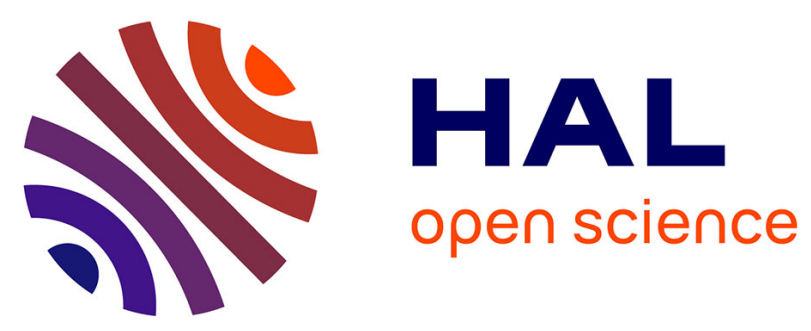

\title{
Analyse des relations de compétition dans une association de luzerne (Medicago sativa L.) et de dactyle (Dactylis glomerata L.) I. Effets sur les dynamiques de croissance en matière sèche \\ Pablo Cruz, Gilles Lemaire
}

\section{To cite this version:}

Pablo Cruz, Gilles Lemaire. Analyse des relations de compétition dans une association de luzerne (Medicago sativa L.) et de dactyle (Dactylis glomerata L.) I. Effets sur les dynamiques de croissance en matière sèche. Agronomie, 1986, 6 (8), pp.727-734. hal-00884930

\author{
HAL Id: hal-00884930 \\ https://hal.science/hal-00884930
}

Submitted on 1 Jan 1986

HAL is a multi-disciplinary open access archive for the deposit and dissemination of scientific research documents, whether they are published or not. The documents may come from teaching and research institutions in France or abroad, or from public or private research centers.
L'archive ouverte pluridisciplinaire HAL, est destinée au dépôt et à la diffusion de documents scientifiques de niveau recherche, publiés ou non, émanant des établissements d'enseignement et de recherche français ou étrangers, des laboratoires publics ou privés. 


\title{
Analyse des relations de compétition dans une association de luzerne (Medicago sativa L.) et de dactyle (Dactylis glomerata L.) I. Effets sur les dynamiques de croissance en matière sèche
}

\author{
Pablo CRUZ \& Gilles LEMAIRE
}

I.N.R.A., Laboratoire d'Agronomie de la Prairie, F 86600 Lusignan

RÉSUMÉ

\begin{abstract}
Nous avons étudié, au sein d'une association luzerne-dactyle et des cultures pures respectives, l'effet cle la compétition sur les dynamiques de croissance en matière sèche des 2 espèces. Le rôle d'un apport d'azote minéral sur les relations de compátition a été également analysé.

La croissance du dactyle associé est fortement réduite par la présence de la luzerne. Cet effet dépressif de la compétition interspécifique sur la graminée est amplifié par la fertilisation azotée, notamment durant la repousse d'été, mettant en évidence la capacité de la légumineuse à concurrencer la graminée pour l'azote minéral du sol. Pour la luzerne, nous avons constaté une valeur de la compétition intraspécifique supérieure à celle de la compétition interspécifique pour tous les niveaux de disponibilité en azote. Pour cette espèce, les fortes vitesses de croissance en période estivale expliquent sa réponse à la fertilisation. A cette période, la culture pure de luzerne à grand écartement répond à la fumure azotée en augmentant sa production en matière sèche de $35 \mathrm{p} .100$, cette augmentation n'étant que de 21 p. 100 dans la structure à écartement normal.

L'azote apporté à l'association est utilisé par l'espèce qui a les taux de croissance les plus élevés en début de repousse. Ceci est le cas de la graminée au printemps et de la légumineuse en été.

L'intérêt agronomique de l'association luzerne-dactyle pour l'économie d'azote est confirmé. La production totale annuelle de la culture pure de dactyle recevant 300 unités d'azote par an est équivalente à celle de l'association non fertilisée et inférieure à celle de l'association recevant 150 unités d'azote par an.
\end{abstract}

Mots clés additionnels : Compétition intraspécifique, compétition interspécifique, fertilisation azotée, Medicago sativa, Dactylis glomerata. increase.

\begin{abstract}
The growth dynamics of lucerne and cocksfoot have been studied in situations of interspecific competition (mixed stands) or intraspecific competition (pure stands). The growth of cocksfoot in association was considerably reduced by lucerne. This effect was amplified by nitrogen fertilization especially during summer regrowth. These results indicate the ability of lucerne to compete with cocksfoot for mineral nitrogen. Competition between lucerne plants was stronger than that with cocksfoot, independent of the level of nitrogen nutrition. For lucerne the very high growth rate in summer explains the response to nitrogen fertilization at this season. This response was higher in stands with wide row spacing, giving an increase of $35 \%$ of dry matter yield, than in slands with narrow row spacing (increase of $21 \%$ ). The nitrogen applied was used by the species which had the higher growth rate at the beginning of regrowth. This was the case of cocksfoot in spring and lucerne in summer. The agronomic value of associations for nitrogen economy was confirmed. The total annual yield of a pure stand of cocksfoot with $300 \mathrm{~kg} \mathrm{~N}$ was equivalent to the yield of the association without $\mathrm{N}$ and inferior to that of the association with $150 \mathrm{~kg} \mathrm{~N}$.
\end{abstract}

Additional key words : Interspecific competition, intraspecific competition, $N$ fertilization, Medicago sativa, Dactylis glomerata.

\section{INTRODUCTION}

Les phénomènes de compétition sont présents dans toutes les communautés végétales naturelles ou cultivées, mono-spécifiques ou pluri-spécifiques. Une situation de compétition est, d'après MALCOLM (1966), définie par l'apparition d'effets dépressifs sur la production des individus mis en présence les uns des autres par rapport à ce qui se passe lorsqu'ils sont isolés. 
L'aspect dynamique des phénomènes de compétition a rarement été étudié comme l'a montré TuRKINGTON (1983). Dans la plupart des travaux, seule une analyse de la production de matière sèche finale a été entreprise ce qui permet une mise en évidence des conséquences de la compétition mais pas une analyse de ses mécanismes.

Dans une association de 2 espèces, il existe à la fois des phénomènes de compétition intraspécifiques et interspécifiques. Le passage de la culture pure à la culture associée implique le remplacement d'une partie de la compétition intraspécifique par une compétition interspécifique et il en va de même de toute modification de la proportion relative des 2 espèces. C'est pourquoi la coexistence de ces 2 types de compétition doit être prise en compte lors des études sur les associations (JACQUARD, 1968 ; TURKINGTON \& HARPER, 1979).

Dans le cas d'une association graminée $\times$ légumineuse, coexistent 2 modes de nutrition azotée, l'assimilation et la fixation. Cette particularité fait que la compétition pour l'azote est difficile à analyser du fait d'un effet très différent d'un apport d'azote sur la compétition intraspécifique et sur la compétition interspécifique.

D’une manière générale, les graminées sont réputées plus compétitives pour l'azote du sol que les légumineuses (HAYNES, 1980). Une telle affirmation est basée principalement sur des études où l'apport d'azote minéral dans l'association a comme conséquence une augmentation de la participation des graminées à la biomasse aérienne (GARDNER et al., 1960 ; NUTALl et al., 1980 ; IONEL et al., 1983 ; HALVOR. SON \& BAUER, 1984).

Dans cette étude, nous avons voulu analyser la compétition entre le dactyle (Dactylis glomerata L.) et la luzerne (Medicago sativa L.) au sein d'une association. L'aspect dynamique de cette compétition a pu être étudié au cours de différentes repousses par le suivi des courbes de croissance de chacune des 2 espèces de l'association et leur comparaison à celles obtenues sur les cultures pures correspondantes. Pour prendre en compte l'effet important de la fertilisation azotée, cette étude a été menée à différents niveaux d'apport d'azote.

Dans ce $1^{\mathrm{er}}$ article, nous présenterons une analyse de la compétition à travers l'étude des dynamiques de croissance en matière sèche des 2 constituants. Dans un $2^{\mathrm{c}}$ article, nous tenterons d'analyser l'effet de la compétition sur la nutrition azotée des 2 espèces.

\section{MATÉRIEL ET MÉTHODES}

\section{A. Principe général de l'étude : nécessité d'analyser séparément les compétitions intra et interspécifi- ques}

Le dispositif expérimental utilisé dans ce travail a été proposé antérieurement par JACQUARD (1968). L'unité de comparaison de la biomasse aérienne est la ligne de semis. D'après cet auteur, la modification de la croissance d'une espèce en association par rapport à sa culture pure n'est pas seulement due à la présence des lignes adjacentes de l'autre espèce mais également à l'absence des lignes de la même espèce. Pour déterminer cet effet de "non présence", il a proposé d'utiliser, outre les cultures pures et associées, les cultures à double écartement de chaque espèce. Ceci permet de comparer les compétitions intra et interspécifiques par leur valeur relative et non par leur valeur absolue.

Nous avons ainsi 3 structures différentes (fig. 1) :

- une ligne de l'espèce A encadrée par des lignes de la même espèce à écartement normal,

- une ligne de l'espèce A encadrée par des lignes du partenaire $B$ à écartement normal,

- une ligne de l'espèce A encadrée par des lignes de la même espèce à double écartement.

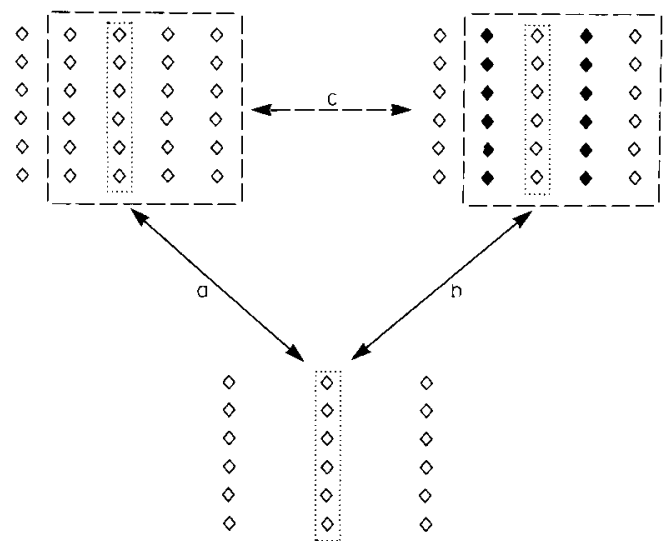

Figure 1

Schéma de semis en ligne permettant d'étudier :

Scheme of the structure of the different stands for comparison of: $a=$ effet de la compétition intraspécifique; effect of intraspecific competition.

$b=$ effet de la compétition interspécifique; effect of interspecific competition.

$c=$ bilan des deux types de compétition; balance between the two types of competition.

- espèce $A$ - espèce B

Les mêmes situations étant réalisées pour l'espèce B.

Ce double écartement (40 cm environ) représente la situation de "non présence " mais il est évident que ces lignes ne sont pas exemptes de la concurrence des lignes voisines, notamment en fin de croissance. La densité de semis de chaque espèce sur la ligne étant la même pour les 3 structures et le fait que les lignes soient exclusivement mono-spécifiques dans tous les traitements minimisent les effets dus à des densités biologiques différentes (ROTILI \& ZANNONE, 1971) à l'intérieur de la ligne.

En plus des comparaisons de biomasses produites par ligne permettant l'analyse des 2 types de compétitions, un tel dispositif permet également d'effectuer des comparaisons de biomasse à l'unité de surface de sol ce qui permet une interprétation à l'échelle agronomique.

\section{B. Protocole expérimental}

Nous avons utilisé la variété de dactyle « Lutetia » en association avec la luzerne "Lutèce ", ces 2 cultivars étant réputés pour avoir un bon comportement en association (GUY, comm. pers.). 
Deux expérimentations ont été conduites. La $1^{\text {re }}$ (Exp. I) a été semée en avril 1981 et a permis d'étudier 4 repousses durant l'année 1982 et la $1^{\text {re }}$ repousse de printemps en 1983. La $2^{\mathrm{e}}$ (Exp. II) a été semée en juin 1983 et a permis d'étudier la $1^{\text {re }}$ repousse (printemps) et la $3^{\text {e }}$ repousse (été) en 1984 . Ces 2 repousses ont donné les résultats les plus contrastés dans l'expérimentation I.

Nous avons établi 3 types de peuplements :

- les cultures pures à écartement normal $(20 \mathrm{~cm}$ entre lignes) $=\mathrm{E}$,

- les cultures pures à double écartement $(40 \mathrm{~cm}$ entre lignes) $=2 \mathrm{E}$,

- les cultures associées en lignes alternées $(20 \mathrm{~cm}$ entre lignes) $=\mathrm{A}$.

Nous avons travaillé à 3 niveaux de nutrition azotée : $0 \mathrm{~N}, 1 / 2 \mathrm{~N}$ et $\mathrm{N}$. Le niveau $\mathrm{N}$ a été ajusté par rapport à une fertilisation non limitante pour le dactyle soit : $120 \mathrm{kgN}$ en début de printemps et $60 \mathrm{~kg}$ pour chacune des repousses suivantes, le niveau $1 / 2 \mathrm{~N}$ correspondant à la moitié de ces apports, soit 60 et $30 \mathrm{~kg}$. Les apports ont été effectués sous forme d'ammonitrate.

Les différents traitements sont représentés sur le tableau 1. On peut noter que toutes les combinaisons possibles n'y figurent pas, ce qui nous aurait conduits à un nombre de parcelles expérimentales trop important pour chacune des 2 expérimentations.

\section{TABLEAU 1}

Traitements effectués dans les deux expérimentations.

Treatments in the two experiments.

\begin{tabular}{|c|c|c|c|c|c|c|c|c|}
\hline & & & & & & & & \\
\hline & & & & Exp. I & & & Exp. I & \\
\hline Peuple & nent & Ligne & $0 \mathrm{~N}$ & $1 / 2 \mathrm{~N}$ & $\mathrm{~N}$ & $0 \mathrm{~N}$ & $1 / 2 \mathrm{~N}$ & $\mathrm{~N}$ \\
\hline Dactyle & pur & $\mathrm{DE}$ & & & + & & & \\
\hline & Dact. & $\begin{array}{l}\text { D2E } \\
\text { DA }\end{array}$ & $\begin{array}{l}+ \\
+\end{array}$ & $\begin{array}{l}+ \\
+\end{array}$ & & + & + & + \\
\hline Association & Luz. & LA & + & + & & + & + & + \\
\hline Luzerne & pure & $\mathrm{LE}$ & + & & & + & + & + \\
\hline Luzerne & pure & $\mathrm{L} 2 \mathrm{E}$ & t & + & & + & + & + \\
\hline
\end{tabular}

Dans notre choix, nous avons délibérément privilégié l'analyse des effets de la compétition sur la luzerne. Nous avons cependant voulu conserver, dans la $1^{\text {re }}$ expérimentation, le dactyle pur à écartement normal $(\mathrm{D}, \mathrm{E}, \mathrm{N})$ comme référence agronomique permettant de comparer la production globale des associations.

Les traitements étaient répartis sur le terrain selon un dispositif en blocs aléatoires complet avec 4 répétitions dans l'expérimentation I et 6 répétitions dans l'expérimentation II.

Le suivi des courbes de croissance était réalisé par des prélèvements hebdomadaires sur chaque bloc de 2 lignes contiguës de $2 \mathrm{~m}$ pour les cultures pures à écartement normal $(E)$ et d'1 ligne de $2 \mathrm{~m}$ pour les cultures pures à double écartement (2E) et pour chaque composant de l'association. La hauteur de coupe était comprise entre 5 et $6 \mathrm{~cm}$.

La nutrition minérale des différents peuplements a été maintenue à un niveau non limitant par des apports en fin d'hiver de 100 unités de $\mathrm{P}_{2} \mathrm{O}_{5}$ et 200 unités de $\mathrm{K}_{2} \mathrm{O}$, le sol étant initialement largement pourvu ( 300 p.p.m. $\mathrm{P}_{2} \mathrm{O}_{5}$ et 250 p.p.m. $\mathrm{K}_{2} \mathrm{O}$ ).

A Lusignan, le sol est un sol brun lessivé sur limon, superposé à une argile rouge à pisolithes. Le pH est de 6,5 environ. Ce sol ne présente aucun facteur pédologique limitant le développement de la luzerne. Seul un déficit hydrique marqué, associé à des fortes ETP, peut, certaines années, limiter fortement la croissance estivale. Dans nos expérimentations, ce déficit a été comblé de manière systématique par irrigation.

\section{RÉSULTATS ET DISCUSSIONS}

\section{A. Comparaison de la production des cultures pures et des associations}

Avant d'analyser la cinétique de croissance des différents peuplements, il nous paraît utile d'effectuer les comparaisons de production obtenue à la fin de chaque repousse.

Le tableau 2 nous permet de comparer la production des 2 cultures pures de références dactyle $\mathrm{E}, \mathrm{N}$ et luzerne $\mathrm{E}, \mathrm{ON}$ avec les cultures associées obtenues lors de l'expérimentation I. On peut noter qu'en absence de tout apport d'azote l'association donne une production totale annuelle équivalente à la moyenne des 2 cultures pures et presque équivalente à celle du dactyle ayant reçu $300 \mathrm{~kg}$ d'azote. Ceci confirme l'intérêt agronomique d'une association luzernedactyle pour l'économie des engrais azotés. Avec un apport de $150 \mathrm{~kg}$ d'azote, l'association est plus productive, non seulement que la moyenne des cultures pures, mais également que le dactyle recevant $300 \mathrm{~kg}$ d'azote. Des résultats analogues avaient été obtenus par PlanCQUAerT $(1976,1982)$ et par Fraser (1982). On peut noter la très faible contribution du dactyle en $3^{\text {e }}$ et $4^{\mathrm{e}}$ repousses, contribution qui n'est pas augmentée par un apport d'azote.

TABLEAU 2

Productions de matière sèche par repousse et totale annuelle des différentes cultures (t. $\left.h a^{-I}\right)$ (1982).

Dry matter yield ( $\left.t . h a^{-1}\right)$ for each regrowth for pure stands and mixtures in 1982.

\begin{tabular}{|c|c|c|c|c|c|}
\hline Repousses & $1^{\text {re }}$ & $2^{e}$ & $3^{e}$ & $4^{e}$ & Total \\
\hline Luz. E & 6,4 & 4,1 & 4,6 & 2,3 & 17,4 \\
\hline Dact. E & 8,0 & 4,5 & 3,7 & 3,5 & 19,7 \\
\hline $\begin{array}{l}\text { Moyennes cultures } \\
\text { pures }\end{array}$ & $\begin{array}{c}7,2 \\
(100)\end{array}$ & $\begin{array}{c}4,3 \\
(100)\end{array}$ & $\begin{array}{c}4,2 \\
(100)\end{array}$ & $\begin{array}{c}2,9 \\
(100)\end{array}$ & $\begin{array}{r}18,6 \\
(100)\end{array}$ \\
\hline Association $1 / 2 \mathrm{~N}$ & & & & & \\
\hline Luzerne & 4,2 & 3,4 & 3,9 & 2,5 & 14,0 \\
\hline Dactyle & 3,8 & 1,8 & 0,7 & 0,6 & 6,9 \\
\hline Total & $\begin{array}{c}8,0 \\
(111)\end{array}$ & $\begin{array}{c}5,2 \\
(121)\end{array}$ & $\begin{array}{c}4,6 \\
(110)\end{array}$ & $\begin{array}{c}3,1 \\
(107)\end{array}$ & $\begin{array}{l}20,9 \\
(112)\end{array}$ \\
\hline Association & & & & & \\
\hline Luzerne & 5,1 & 3,1 & 3,2 & 2,1 & 13,5 \\
\hline Dactyle & 3,1 & 1,3 & 0,6 & 0,6 & 5,6 \\
\hline Total & $\begin{array}{c}8,2 \\
(114)\end{array}$ & $\begin{array}{c}4,4 \\
(102)\end{array}$ & $\begin{array}{c}3,8 \\
(90)\end{array}$ & $\begin{array}{c}2,7 \\
(93)\end{array}$ & $\begin{array}{c}19,1 \\
(103)\end{array}$ \\
\hline
\end{tabular}




\section{B. Comparaison des dynamiques de croissance en cul- tures pures et associées}

\section{Dynamique de croissance du dactyle}

Le protocole de l'expérimentation I ne nous permet pas d'analyser simultanément les effets de la compétition intraspécifique et de la compétition interspécifique sur la dynamique de croissance du dactyle dans l'association. Les courbes de la figure 2 mettent en évidence l'effet de la compétition de la luzerne sur le dactyle à 2 niveaux de nutrition azotée. Comme dans toutes les cultures pures, nous présentons seulement les courbes correspondantes aux repousses de printemps ( $1^{\text {re }}$ repousse) et d'été ( $3^{\mathrm{e}}$ repousse) car elles représentent les situations les plus contrastées. Pour chacune des 2 repousses, on constate que la diminution de croissance du dactyle par le voisinage de la luzerne est toujours beaucoup plus marquée lorsqu'il y a eu un apport d'azote et ceci est perceptible dès le début de la repousse. Le tableau 3 exprime l'effet dépressif de la luzerne sur la production du dactyle à la fin de chaque repousse en valeur relative selon la méthode proposée par JACQUARD (1968).

La figure 2 nous permet également une analyse de la cinétique de réponse du dactyle à un apport d'azote
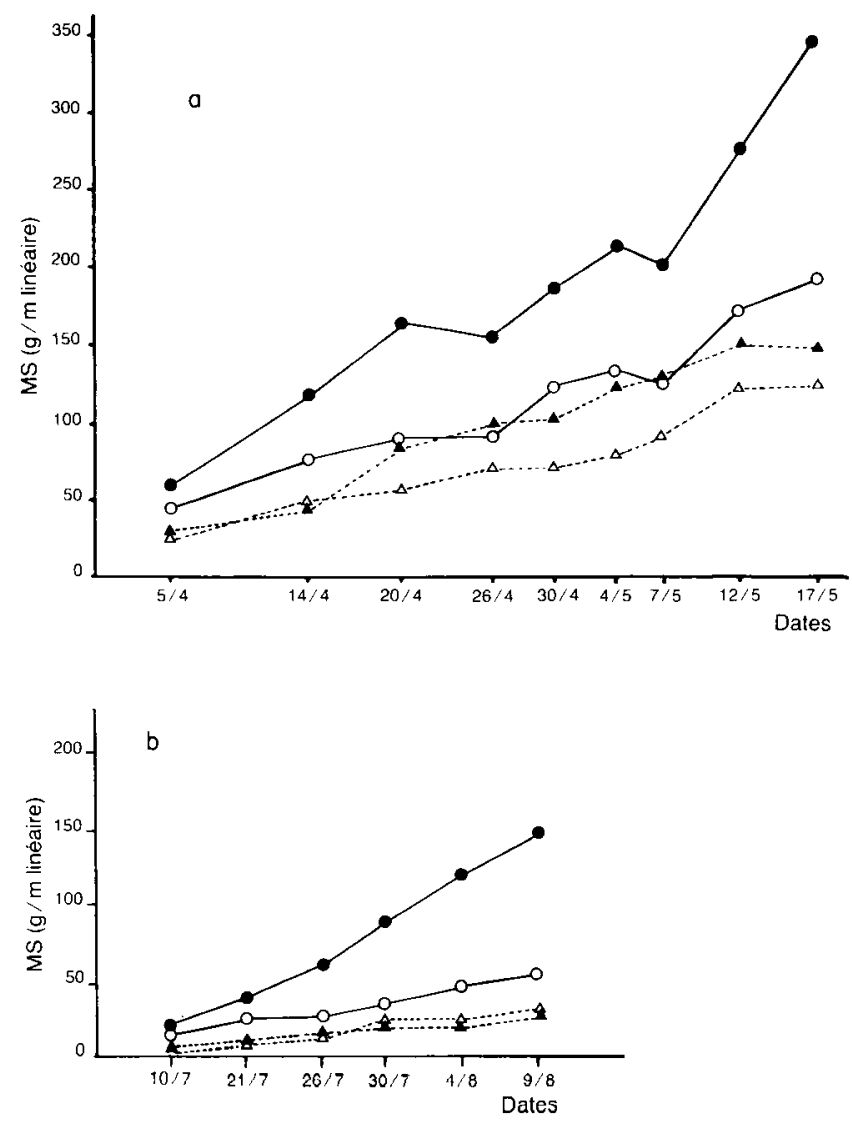

Figure 2

Courbes de croissance en matière sèche de lignes de dactyle (1982).

Dry matter growth curves for cocksfoot rows in 1982.

a) = repousse de printemps; spring regrowth. b) = repousse d'été ; summer regrowth.

- dactyle pur à double écartement, $N$; pure stand double-spaced row, $N$.

dactyle pur à double écartement, ON; pure stand double-spaced row, 0 .

$\Delta$ dactyle associe, $N$; cocksfoot in mixture, $N$.

$\triangle$ dactyle associé, $O N$; cocksfoot in mixture, $O N$.

\section{TABLEAU 3}

Indice de compétition interspécifique (Ie) sur le dactyle associé. Index of interspecific competition (Ie) for cocksfoot in mixture.

$I e=\frac{M S D_{a}-M S D_{2 e}}{M S D_{2 e}} \times 100$,

où $M S D_{a}=$ matière sèche du dactyle associé à la luzerne; dry matter yield of cocksfoot in mixture.

$M S D_{2 e}=$ matière sèche du dactyle double écartement; dry matter yield of cocksfoot in pure stand with double row spacing.

\begin{tabular}{rcccc}
\hline & $1^{\text {er }}$ cycle & $2^{\mathrm{e}}$ cycle & $3^{\mathrm{e}}$ cycle & $4^{\mathrm{e}}$ cycle \\
\hline $0 \mathrm{~N}$ & -36 & -35 & -37 & -61 \\
$1 / 2 \mathrm{~N}$ & -57 & -57 & -81 & -84 \\
\hline
\end{tabular}

dans les 2 types de peuplement : culture pure à double écartement et association. On constate une réponse très marquée en culture pure dès le début de la repousse, ce qui aboutit à une augmentation de production d'environ 100 p. 100. Dans l'association, la réponse à l'apport d'azote reste faible en $1^{\text {re }}$ repousse où elle tend rapidement à se stabiliser (courbes parallèles) ; elle est cependant nulle en $3^{\mathrm{e}}$ repousse malgré la très forte réponse du dactyle en culture pure à cette période. On peut donc attribuer cette absence de réponse du dactyle associé à l'apport d'azote à la présence de la luzerne.

\section{Dynamique de croissance de la luzerne}

Pour cette espèce, le protocole nous permet de comparer les valeurs relatives de la compétition intraspécifique et de la compétition interspécifique sur la cinétique de croissance de la ligne de luzerne. Cette comparaison ne peut en réalité s'effectuer qu'en absence d'apport d'azote $(0 \mathrm{~N})$ dans l'expérimentation I. La figure 3 montre que, aussi bien au printemps qu'en été, la croissance d'une ligne de luzerne est davantage déprimée par la présence de lignes adjacentes de la même espèce que par celle de lignes de dactyle. Ceci est entièrement confirmé par les résultats de l'expérimentation II réalisée à un niveau de nutrition azotée élevée (N) (fig. 4). Ainsi il est intéressant de montrer que, même lors des repousses de printemps avec une forte fumure azotée, l'agressivité du dactyle vis-à-vis de la luzerne reste nettement inférieure à celle de la luzerne vis-à-vis d'elle-même, malgré les capacités de croissance de la graminée nettement supérieures à cette période. On peut donc penser que l'intensité de la compétition n'est pas liée directement à la capacité de croissance en matière sèche des 2 espèces mais à leur capacité d'interception de la lumière qui est déterminée à la fois par la dynamique d'expansion de l'indice foliaire et par la géométrie du feuillage (VARLET-GRANCHER \& BONHOMME, 1979).

\section{Analyse de la réponse de la luzerne à l'apport d'azote}

La comparaison des effets de l'azote sur la cinétique de croissance de la luzerne en culture pure (L, 2E) ou en association (A) peut être faite à partir des courbes des figures 5 et 6 . Dans l'expérimentation I 

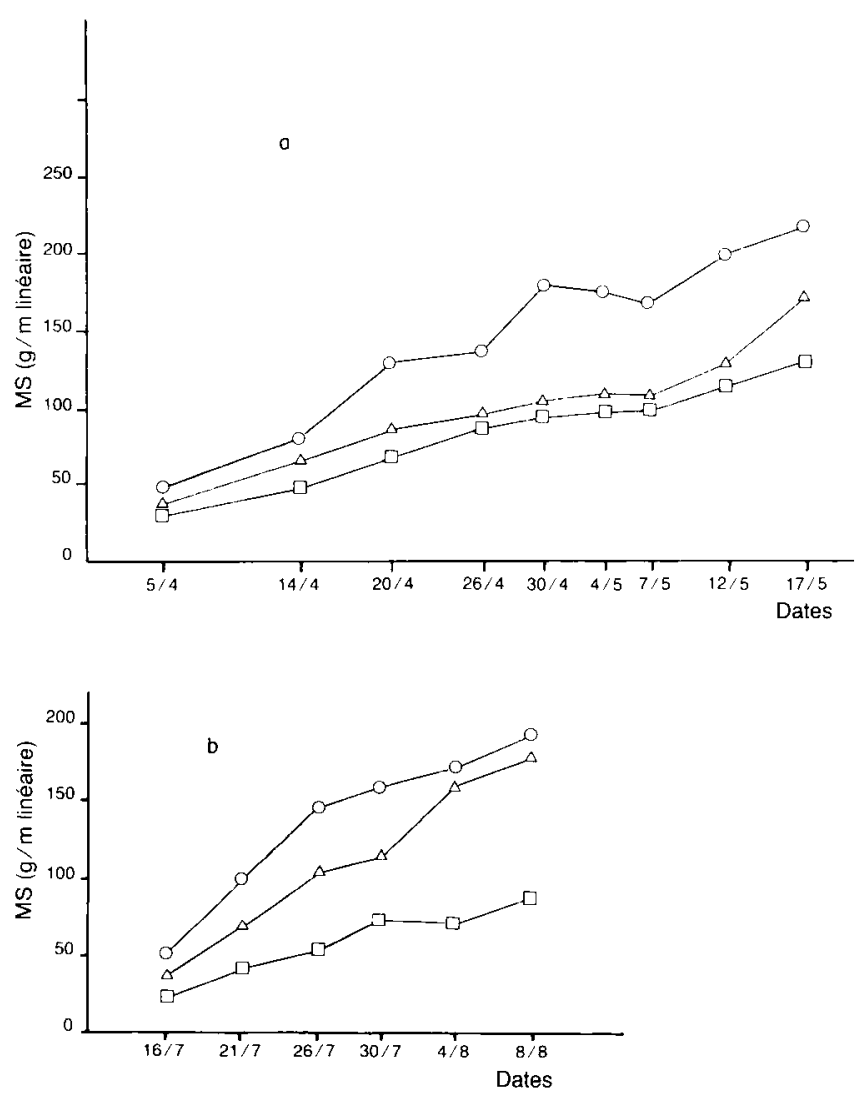

Figure 3

Courbes de croissance en matière sèche de lignes de luzerne (1982). Dry matter growth curves for lucerne rows in 1982.

a) = repousse de printemps; spring regrowth. b) = repousse d'été ; summer regrowth.

luzerne double écartement, oN ; pure stand double-spaced row, oN.

$\triangle$ luzerne associée, oN; mixture, $0 N$.

$\square$ luzerne écartement normal, oN ; pure stand single-spaced row, ON.

(fig. 5), l'apport d'azote sur la luzerne pure à double écartement ( $\mathrm{L}, 2 \mathrm{E})$ produit au printemps une accélération de la croissance jusqu'au début du mois de mai, suivie par un effet dépressif assez net en fin de repousse. L'apport d'azote sur l'association produit un effet dépressif sur la croissance de la luzerne qui ne se manifeste qu'à partir de la fin du mois d'avril. Par contre, en été, on constate un effet positif très net de l'apport d'azote sur la croissance de la luzerne pure qui se manifeste dès le début de la repousse. De même, il existe une réponse importante de la luzerne associée, principalement en fin de repousse, de telle sorte que sa production devient presque égale à celle de la luzerne pure.

Dans l'expérimentation II (fig. 6), nous confirmons globalement le comportement des cultures pures de luzerne à double écartement $(\mathrm{L}, 2 \mathrm{E})$ vis-à-vis d'un apport d'azote :

- absence de réponse marquée au printemps (effet légèrement favorable en début de croissance, suivi par un effet dépressif),

- forte réponse à l'azote en été.

De plus, ces nouvelles données montrent que la réponse à l'azote de la ligne de luzerne en culture dense (L, E) est inférieure à celle observée en culture à
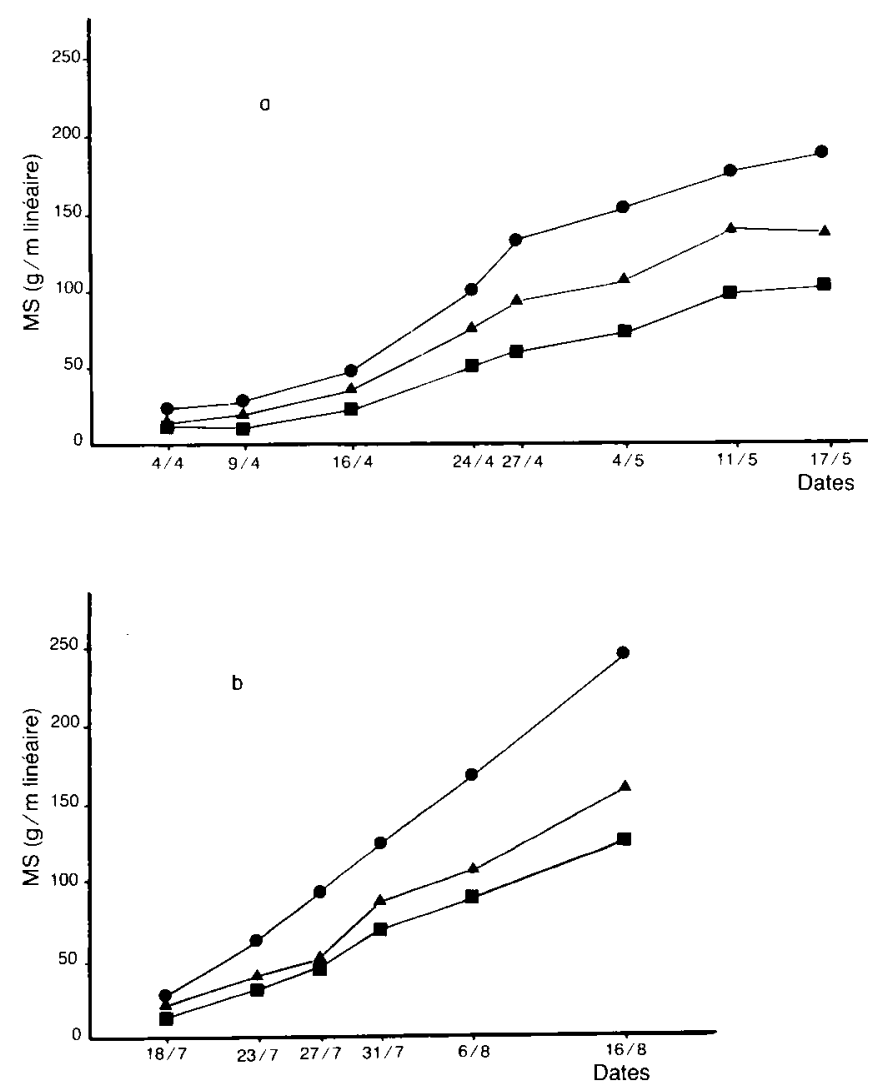

Figure 4

Courbes de croissance en matière sèche de lignes de luzerne (1984). Dry matter growth curves for lucerne rows in 1984.

a) = repousse de printemps; spring regrowth. b) = repousse d'été ; summer regrowth.

- luzerne double écartement, $N$; pure stand double-spaced row, $N$.

A luzerne associée, $N$; mixture, $N$.

luzerne écartement normal, $N$; pure stand single-spaced row, $N$.

double écartement (L, 2E). Le tableau 4 nous permet d'étudier les différences de production exprimée en $\mathrm{t} / \mathrm{ha}$, obtenues en fin de repousse pour les 3 niveaux d'apport d'azote $0 \mathrm{~N}, 1 / 2 \mathrm{~N}$ et $\mathrm{N}$. Nous constatons que l'apport d'azote augmente la production à l'ha de 35 p. 100 sur la luzerne à double écartement et de seulement 21 p. 100 pour l'écartement normal. Enfin, à fort niveau d'azote, la production des 2 peuplements devient équivalente. On peut interpréter ces résultats de la manière suivante : en peuplement dense, c'est surtout la lumière, donc la nutrition carbonée, qui serait le facteur le plus limitant de la croissance, d'où une plus faible réponse à l'azote ; à grand écartement

\section{TABLEAU 4}

Production de matière sèche des cultures pures de luzerne (t. $\left.h a^{-1}\right)$. Repousse d'été 1984.

Dry matter yields of pure stands of lucerne in summer 1984 $\left(t . h a^{-1}\right)$.

\begin{tabular}{lccc}
\hline \hline & $0 \mathrm{~N}$ & $1 / 2 \mathrm{~N}$ & $\mathrm{~N}$ \\
\hline Luzerne écart. normal & 5,3 & 6,0 & 6,4 \\
Luzerne double écart. & 4,6 & 5,4 & 6,2 \\
\hline
\end{tabular}



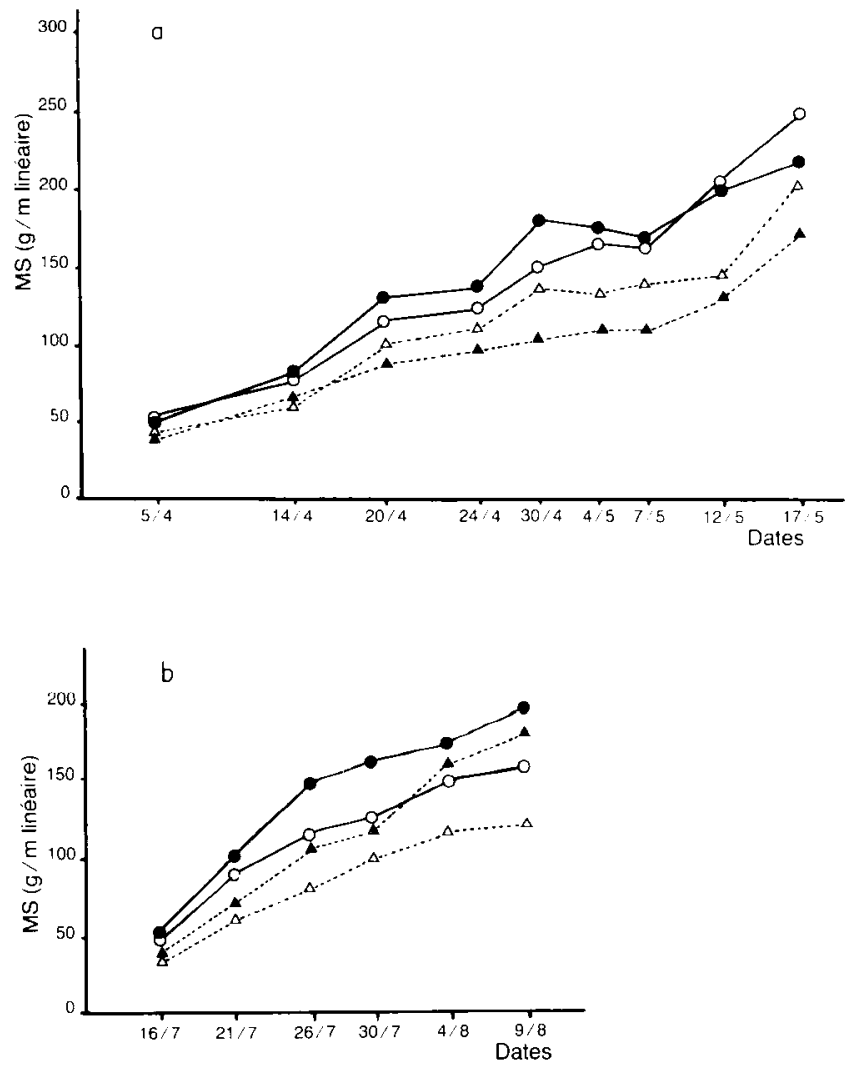

Figure 5

Courbes de croissance en matière sèche de lignes de luzerne (1982). Dry matter growth curves for lucerne rows in 1982.

a) = repousse de printemps; spring regrowth. b) = repousse d'été ; summer regrowth.

- luzerne double écartement, $N$; pure stand double-spaced row, $N$. luzerne double écartemeni, $0 N$; pure stand double-spaced row, $0 N$.

A luzerne associée, $N$; mixture, $N$.

$\triangle$ luzerne associée, ON; mixture, $0 N$.

un apport d'azote permettrait la valorisation du supplément de carbone disponible (meilleur éclairement), d'où la compensation du déficit de production engendré par la plus faible densité de peuplement.

Sur la luzerne associée, nous obtenons par contre en 1984 (Exp. II) des comportements différents de ceux obtenus en 1982 (Exp. I) : absence d'effet dépressif de l'apport d'azote au printemps, absence d'effet positif en été. Les conditions de printemps moins favorables à la croissance en 1984 (froid et sec) n'auraient pas permis au dactyle d'avoir une réponse suffisante à l'apport d'azote en association pour provoquer un effet dépressif sur la luzerne.

La figure 7 nous permet d'analyser de manière plus complète les différences de comportement, en été, de la luzerne associée vis-à-vis de l'apport d'azote dans les 2 expérimentations. On constate pour les 2 années un comportement totalement inversé des vitesses de croissance de la luzerne et du dactyle associés (fig. $7 a$ et $7 b)$; malgré cela, la luzerne pure a maintenu une réponse équivalente à l'azote (fig. $7 c$ ).

On remarque cependant, pour une même date de coupe, un net décalage du départ en croissance de la luzerne en 1984, que ce soit en culture pure ou en association. Les conditions climatiques des 2 années ne permettent pas d'expliquer ces différences (nous rappelons que les parcelles étaient irriguées). Par con-
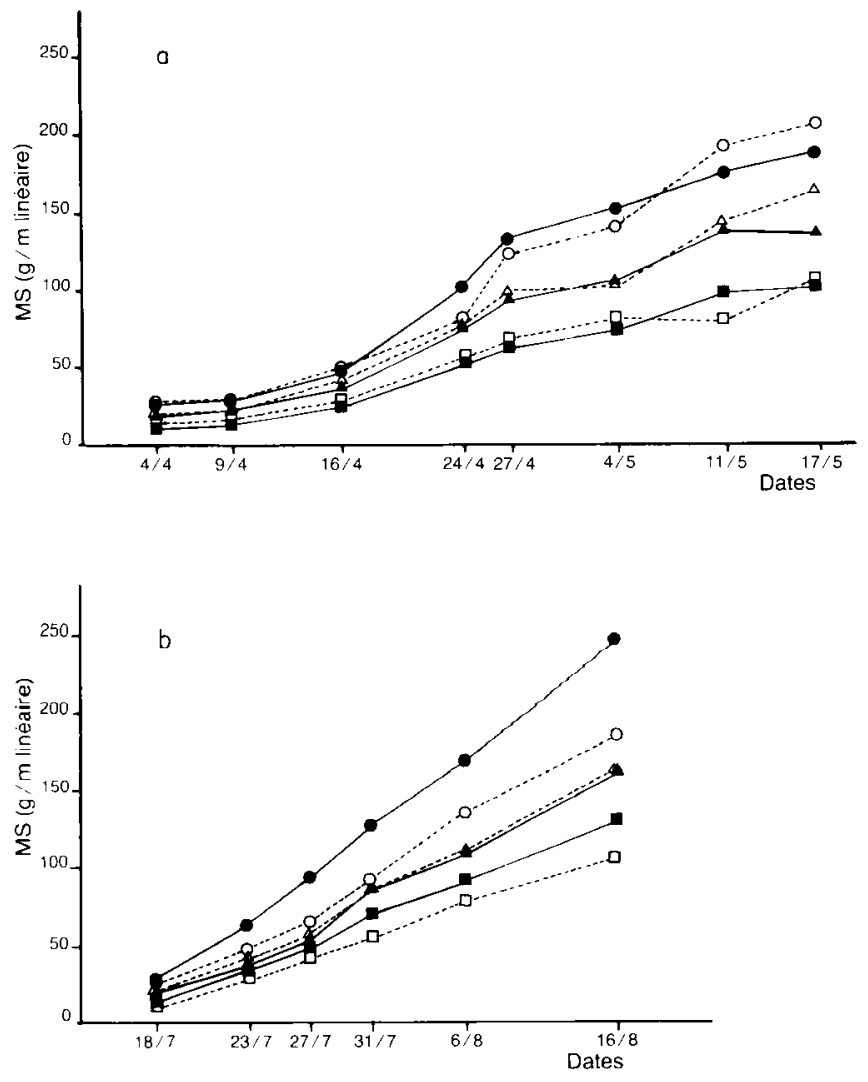

Figure 6

Courbes de croissance en matière sèche de lignes de luzerne (1984). Dry matter growth curves for lucerne rows in 1984.

a) = repousse de printemps; spring regrowth. if) $=$ repousse d'été ; summer regrowth.

\begin{tabular}{ll}
$\left.\begin{array}{l}\text { luzerne double écartement } \\
\text { pure stand double-spaced row } \\
\text { luzerne associée } \\
\text { mixture } \\
\text { luzerne écartement normal } \\
\text { pure stand single-spaced row }\end{array}\right\} \begin{cases}\bullet & N \\
0 & 0 N\end{cases}$ \\
$\begin{array}{ll}\Delta & N \\
\triangle & O N\end{array}$ \\
\hline $\begin{array}{ll} & N\end{array}$
\end{tabular}

tre, nous nous sommes aperçu que, par erreur, en 1984, la coupe du début juillet avait été réalisée à une hauteur très faible $(2 \mathrm{~cm}$ environ) par rapport à la hauteur habituelle $(5-6 \mathrm{~cm})$. Compte tenu de la sensibilité particulière de la luzerne à la hauteur de coupe, on trouve là une explication plausible à son retard de croissance. Le dactyle, par contre, comme l'ensemble des graminées, s'est montré peu sensible à la hauteur de coupe et a pu ainsi bénéficier du retard de croissance de la luzerne et réagir positivement à l'apport d'azote.

Ainsi, cette erreur expérimentale nous permet de mettre en évidence le fait que ce sont les différences relatives de croissance en début de repousse qui conditionnent la réponse de chacune des 2 espèces associées à l'apport d'azote. Tout facteur contribuant à ralentir préférentiellement la vitesse d'établissement de la surface foliaire de l'une des 2 espèces (hauteur de coupe dans le cas présent, mais éventuellement déficit hydrique, nutrition phospho-potassique, fréquence d'exploitation, etc...) aura comme résultat une augmentation de la réponse à l'azote de l'autre espèce dans l'association.

Ainsi, un apport d'azote visant à rééquilibrer l'asso- 

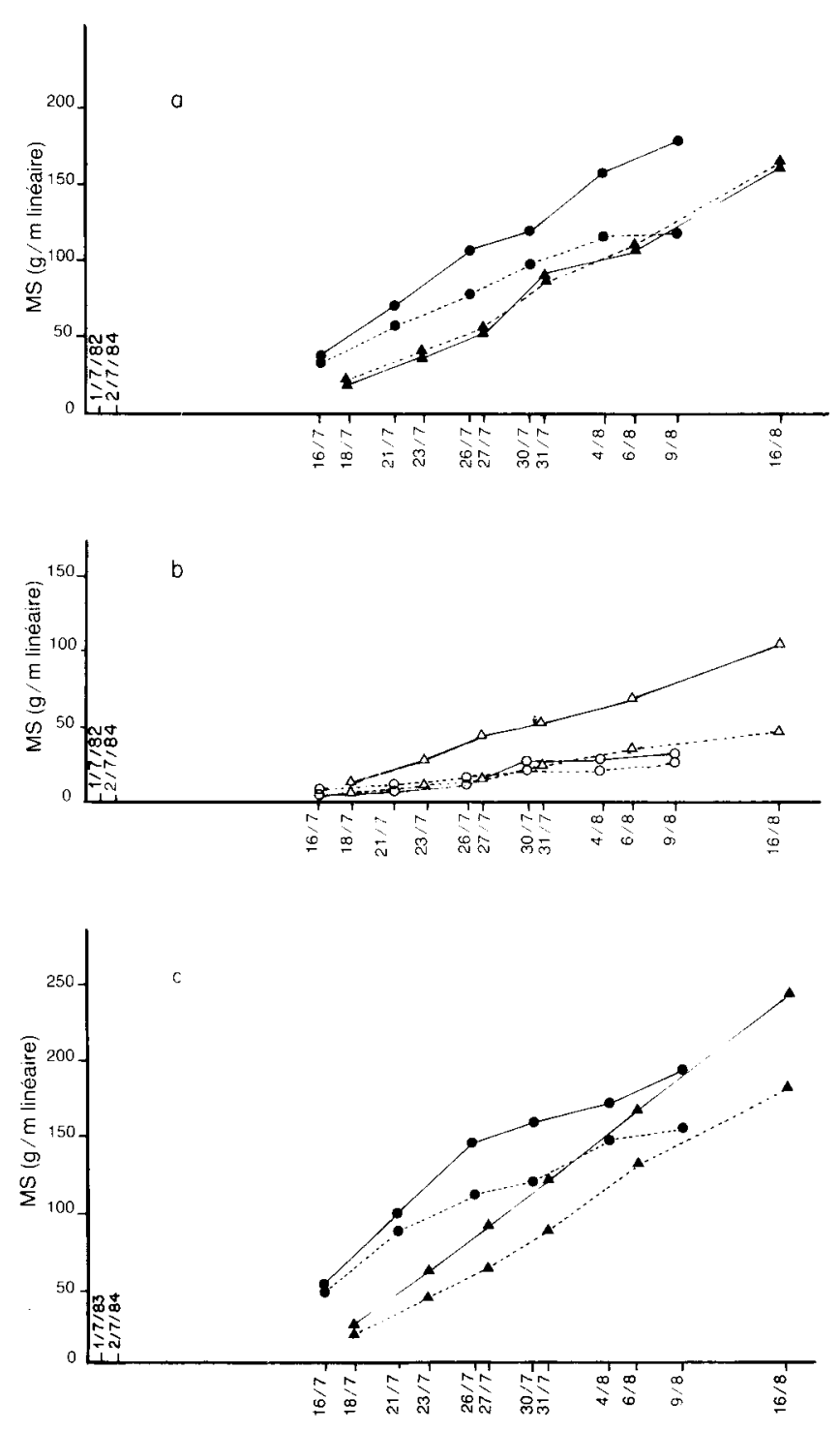

Figure 7

Courbes de croissance en matière sèche.

Dry matter growth curves.

a) lignes de luzerne associée

b) lignes de coctle associe 1984 b) lignes de dactyle associé $\int 01982$ row of cocksfoot in mixture $\triangle 1984$

c) lignes de luzerne double écartement; double-spaced row of lucerne

ciation au bénéfice de la graminée durant la période estivale peut aboutir à l'inverse de l'effet recherché si, comme en 1982, la capacité de repousse de la luzerne en début de cycle n'est pas limitée par un autre facteur. Cette interprétation pourrait permettre d'expliquer les résultats contradictoires d'un apport d'azote sur les associations à base de luzerne tels qu'ils ont été signalés par JACQUARD (1977).

La forte capacité de réponse de la luzerne à un apport d'azote minéral pendant la période estivale est en accord avec les résultats obtenus par certains auteurs qui signalent une réponse importante de cette espèce dans les périodes de très forte croissance (HOGLUND et al., 1974 ; WERY, 1983 ; REHM et al., 1975). En période estivale et en conditions irriguées, les vitesses de croissance mesurées sur la luzerne étaient de l'ordre de $192 \mathrm{~kg}$ de MS . ha-1 $\cdot \mathrm{j}^{-1}$ alors qu'au printemps elles n'étaient que de $99 \mathrm{~kg}$ MS . ha $\mathrm{a}^{-1} \cdot \mathrm{j}^{-1}$, ce qui correspond à des besoins instantanés en azote de $3 \mathrm{~kg} \cdot \mathrm{ha}^{-1} \cdot \mathbf{j}^{-1}$ au printemps à $6 \mathrm{~kg} \cdot \mathrm{ha}^{-1} \cdot \mathrm{j}^{-1}$ en période estivale. On peut supposer, dans ces conditions, que la seule fixation d'azote ne puisse satisfaire une telle demande.

\section{CONCLUSION}

Les résultats mis en évidence dans cette étude nous ont permis de montrer que la dynamique de croissance en matière sèche de chacun des 2 partenaires d'une association ne constitue pas en soi un élément déterminant la compétition interspécifique, mais apparaît plutôt comme une résultante de la différence de dynamique d'interception de la lumière entre les 2 espèces en début de repousse. A cette étude dynamique de la croissance en matière sèche devrait être couplée une étude dynamique de l'expansion de l'indice foliaire et de l'interception de la lumière des 2 espèces associées. Cependant, les outils nécessaires à de telles mesures et à leur interprétation, sous forme de modèles développés par ailleurs dans des couverts mono-spécifiques relativement homogènes (GOSSE et al., 1982), ne sont pas encore à l'heure actuelle adaptés aux études sur des couverts anisotropes tels que les associations en lignes alternées.

Ces résultats ont également mis en évidence le rôle primordial de la nutrition azotée dans les relations de compétition entre la graminée et la légumineuse. Cependant, il apparaît que l'effet relatif d'un apport d'azote sur la compétition entre les 2 espèces est fortement déterminé par les conditions générales de croissance pouvant orienter la compétition dans un sens ou dans l'autre.

Une étude plus spécifique de la compétition entre les 2 espèces pour l'utilisation de l'azote minéral du sol est indispensable ; elle sera développée dans l'article suivant.

Reçu le 29 novembre 1985. Accepté le 18 avril 1986.

\section{RÉFÉRENCES BIBLIOGRAPHIQUES}

Fraser T. J., 1982. Evaluation of "grasslands Matua" prairie grass and "grassland Maru" Phalaris with and without lucerne in Canterbury. N.Z.J., exp. Agric., 10 (3), 235-237.

Gardner E. H., Jakson T. L., Webster G. R., Turley R. H., 1960. Some effects of fertilization on the yield and botanical and chemical composition of irrigated grass-clover pasture. Can. J. Plant Sci., $40,546-562$.
Gosse G., Chartier M., Varlet-Grancher C., Bonhomme R., 1982. Interception du rayonnement utile à la photosynthèse chez la luzerne : variation et modélisation. Agronomie, 2 (6), 583-588.

Halvorson G. A., Bauer A., 1984. Yield and botanical composition of a grass-legume mixture on reclaimed land as affected by nitrogen and phosphorus fertilizer. Agron. J., 76 (3), 355-362. 
Haynes J., 1980. Competitive aspects of the grass-legume association. Adv. Agron., 33, 227-261.

Hoglund J. H., Doughery C. T., Langer R. H. M., 1974. Response of irrigated lucerne to defoliation and nitrogen fertilizer. N.Z.J. exp. Agric., 2, 7-11.

Ionel A., Cazaceanu A., Cazaceanu I., 1983. The effect of fertilizer on production and structure of plant cover on temporary pasture. Rev. Cresteres Anim., 33 (9), 1-5.

Jacquard P., 1968. Manifestation et nature des relations sociales chez les végétaux supérieurs. Oecol. Planta, II, 137-168.

Jacquard P., 1977. Relations entre espèces dans les associations graminées-légumineuses. Sel. $f r ., 24,3-28$.

Malcolm W. N., 1966. Biological interactions. Bot. Rev., 32 (3), 243-254.

Nutall W. F., Cooke D. A., Waddington J., Robertson J. A., 1980

Effect of nitrogen and phosphorus fertilizers on a bromegrass and alfalfa mixture grown under two systems of pasture management. I. Yield, percentage legume in sward and soil tests. Agron. J., 72 (2), 289-294.

Plancquaert Ph., 1976. Résultats expérimentaux de l'I.T.C.F. concernant les associations graminées-légumineuses. Fourrages, 66, 31 62 .
Plancquaert Ph., 1982. La luzerne et le trèfle violet en culture pure ou en association : aspects phytotechniques. Fourrages, 90, 135159 .

Rehm G. W., Nichols J. T., Sorensen R. C., Moline W. J., 1975 Yield and botanical composition of an irrigated grass-legume pasture as influenced by fertilization. Agron. J., 67 (1), 64-68.

Rotili P., Zannone L., 1971. L'impiego della competizioni nel miglioramento genetico dell'erba medica. Quad. Sper., 3, 72-86.

Turkington R., 1983. Leaf and flower demography of Trifolium repens L. I. Growth in mixture with grasses. New Phytol., 93, 599616.

Turkington R., Harper J. L., 1979. The growth, distribution and neighbouring relationships of Trifolium repens in a permanent pasture. II. Inter and intra-specific contact. J. Ecol., 67, 219-230.

Varlet-Grancher C., Bonhomme R., 1979. Application aux couverts végétaux des lois de rayonnement en milieu diffusant. Il. Interception de l'énergie solaire par une culture. Ann. agron., 30 (1), 1-26.

Wery J., 1983. Contribution à l'étude de la nutrition azotée d'une légumineuse fourragère (Medicago sativa L.) et de légumineuses à graines. Thèse Doct. Ing. Ec. nat. sup. agron. Montpellier, 239 p. 